\title{
Sleep deprivation in healthcare professionals and medical errors: how to recognize them?
}

\author{
Keywords: sleep deprivation, risk, healthcare professionals, \\ medical errors, night shifts
}

\author{
Abbrevations: SD, sleep deprivation; RTA, risk-taking \\ attitudes; VTA, ventral tegmental area
}

\section{Opinion}

It is common finding that healthcare professionals, especially nurses and doctors, might incur frequent night shifts, on-calls and stress that will lead to loss of sleep, and manifest symptoms of sleep deprivation (SD). Our working hypothesis is that healthcare professionals who start to reduce their hours of physiological sleep are more likely to underestimate the risks linked to any action performed in their clinical practice and to make more errors. Extensive research confirms that SD affects functioning and concentration of physicians. ${ }^{1}$ It is also reported that allowing SD to permeate all medical specialties will cause damage both to patients and healthcare professionals. ${ }^{2}$ Errors reported in nurses affected by SD are, an incorrect characterization of a patient's problem, neglected medication, neglected care, mistakes in the communication of doctor's instructions, mistakes in recording events, and gaps in treatments when needed. ${ }^{3}$ The authors of the current study, all physicians practicing in a county hospital in general adult psychiatry, believe that sleep-deprived nurses and doctors are more likely to commit medical errors as risk-taking attitudes (RTA) increase directly with the number of hours of lost sleep (NHLS): RTA $=k$ NHLS (with $k=$ constant).

This direct correlation emerging from the previous equation will impact on the legal liability of healthcare workers when not able to allocate protected time for their sleep. Sadly, some nurses and doctors, for economic and personal reasons, decide to give up their sleep hours in exchange of increased salaries, better job opportunities or, simply, for achieving appreciation from their managers. In any case, risk assessment by sleep-deprived professionals becomes faulty when tiredness increases as clear judgment decreases.

In our pilot study (unpublished), we found that after sleepdeprivation, medical errors become more common, especially for clinical practice performed during night shifts, while nurses and doctors working in these turns are more likely to become victims of professional investigations and a loss of qualified accountability. Major cognitive errors that we found in nurses and doctors victims of chronic sleep deprivation are, omissions (actions are not fully completed), generalizations (similar data are considered equal), unawareness of procedural policies (as most of them are available only at day time), incomplete clinical and psychiatric assessment, missed patients feedback, reduced critical judgement and diagnostic wisdom, prescription errors (medications might have the wrong dosage or might not be indicated for the ongoing pathology), misinterpretations (automatisms are preferred to critical thinking), and missed steps due to urgency.

These data shed light on the fact that even trained nurses and doctors, under sleep deprivation, make a summative and generalized evaluation of clinical data and risks while missing details and collateral history of their patients. In fact, tiredness will reinforce professional

\author{
Volume 2 Issue I - 2018
}

\section{Carlo Lazzari Ahmed Shoka, Basavaraja Papanna, Georgios Mousailidis}

Essex Partnership University Foundation Trust, United Kingdom

Correspondence: Carlo Lazzari, Kingswood Centre, Turner Road, Colchester, CO4 5JY, United Kingdom, Tel 00447939919992, Email carlolazzari2015@gmail.com

Received: January 23, 2018 | Published: February 08, 2018

automatism with the reduction of differentiation and critical thinking about correct actions towards individual patients. Another aspect observed in SD is increased irritability that reduces a collaborative practice while restricting the acceptance of divergences in opinion in the team. Also, errors linked to SD are frequent in locum doctors who cover shifts in hospitals and premises they are not familiar with. In these cases, lack of knowledge of basic policies in novel environments, unawareness of local procedures, and misinformation about team priorities in unfamiliar wards, and misuse of unapproved strategies and skills will increase the likelihood of medical errors. An additional consequence of SD is on the character of healthcare professionals. Although many lament depression, irritability, and anxiety, others, equally describe improved affect with decreased fear, heightened mood and a deceitful increase in self-esteem. Besides, SD can be a consequence of increased stress and not merely an effect of night shifts.

Neural mechanisms underlying cognitive errors during SD are not entirely known. However, we hypothesize that the mesolimbic pathway might be involved. A reduction in dopamine transmission will be responsible for demotivation, loss of pleasure, reduced skilled regulation of muscular activities, while inhibitory activities of frontal lobes become less prevalent with consequent impulsivity and irritability. Moreover, a reduction in serotonin transmission will result in depression or hypomania, sleep-wake variations, altered working memory, and faulty cognitive operations. Additionally, dopaminergic pathways that start from the ventral tegmental area (VTA) are critical components in the neural messages that control excitement, impulses, and rewards. ${ }^{4}$

Croskerry $^{5}$ provides a detailed description of cognitive errors in medicine while advocating the use of a self-reflective practice or 'stepping back' from the direct dilemma to analyze and ponder the own thoughts. ${ }^{5}$ Another strategy to reduce errors during SD involves a whole inter professional team tackling a problem, taking time out to reflect on it, and finally reaching a joint decision for a possible action. ${ }^{6}$

\section{Conclusion}

The current article summarizes a vital consequence of sleep deprivation in health professionals. The occurrence of medical errors due to sleep deprivation is likely to put patients at risk and health workers under investigation on their professional liability. Therefore, awareness of medical errors under SD should accompany healthcare 
policies especially when health professionals have not an immediate backup.

\section{Acknowledgements}

No funds were received by the author for the current article.

\section{Conflict of interest}

The author declares no conflict of interest for the current article.

\section{References}

1. Eastman P. Does sleep deprivation increase medical errors? Emergency Medicine News. 2003;25(9):48-49.

2. Sugden C, Aggarwal R, Darzi A. Re: Sleep deprivation, fatigue, medical error and patient safety. Am J Surg. 2010;199(3):433-434.
3. Ramadan MZ, Alsaleh SK. The association of sleep deprivation on the occurrence of errors by nurses who work the night shift. Curr Health Sci J. 2014;40(2):97-103.

4. Margolis EB, Lock H, Chefer, et al. Kappa-opioids selectively control dopaminergic neurons projecting to the prefrontal cortex. P Natl Acad Sci USA. 2006;103(8):2938-2942.

5. Croskerry P. The importance of cognitive errors in diagnosis and strategies to minimize them. Acad Med. 2003;78(8):775-780.

6. Lazzari C, Masiello I, Shoka A. Chapter 1. Theoretical Models of Social Mind and Social Thinking in Interprofessional Education. In: Nata RV editor. Progress in Education, New York, USA: Nova Publisher; 2007;48:1-38. 